\title{
The Intelligent Texture Anti-counterfeiting Algorithm Based on DCT
}

\author{
Yicheng Li, Yujia Li, Jingbing Li* \\ College of Information Science and Technology, Hainan University, Haikou, 570228, China \\ Email: Jingbingli2008@hotmail.com
}

\begin{abstract}
This paper put forward an algorithm based on DCT and human visual characteristics for the identification of texture anti-counterfeiting tags. Firstly, the method extracted the visual feature vectors of texture image through DCT, and then created a database of texture images' feature vectors. Compare the Normalized Cross-correlation (NC) between the feature vectors of texture tags. The results of experimental study demonstrate that the proposed algorithm has strong robustness against common and geometric attacks. In addition, this algorithm can reduce the database storage space occupied and has better practicability and convenience by comparing with the existing texture anti-counterfeiting technology.
\end{abstract}

Keywords - texture anti-counterfeiting tag; feature vector; DCT; strong robustness

\section{INTRODUCTION}

In recent years, counterfeiting caused serious harm to the world economy and consumers ${ }^{[1]}$. Texture anti-counterfeiting is one kind of new anti-counterfeiting technology developed in recent years. It's a collection of information queries, database storage, digital printing and other technologies in one. In addition, there are many advantages of texture anticounterfeiting, Such as random selection, difficult to counterfeit, easy to identify, widely applicable, long-term and effective $^{[2]}$. Texture anti-counterfeiting is an anticounterfeiting technology that uses packaging material itself inherent speckle mark as anti-fake identifying marker ${ }^{[3]}$. Therefore, texture anti-counterfeiting research and development become a new anti-counterfeiting technology in recent years.

Although technical advantages are obvious, but it still have some shortcomings. For example, identification is difficult to educate consumers to verify and distinguish, and it need to enter the serial number query ${ }^{[4]}$. Although the two-dimensional code scanning don't need to enter the serial number ${ }^{[5]}$, but in the case of poor light, the identification is more difficult and it takes a long time.

Currently, identification of security and intelligence about the texture does very little. Inspired by Kutter and Kaewkammerd extracted the visual feature by wavelet transform ${ }^{[6,7]}$. We propose an algorithm based on DCT. Acquire the visual feature vectors of the texture image by using DCT, and compare the feature vector of texture-tag with the feature vectors in database of the $\mathrm{NC}^{[7-12]}$. The experiment results showed that the algorithm has good robustness against common and geometrical attacks.

\section{THE FUNDAMENTAL THEORY}

\section{A. The discrete Cosine transform (DCT)}

Two-dimensional discrete cosine transformation formula is as follows:

$$
\begin{gathered}
F(u, v)=c(u) c(v) \sum_{x=0}^{M-1} \sum_{y=0}^{N-1} f(x, y) \cos \frac{\pi(2 x+1) u}{2 M} \cos \frac{\pi(2 y+1) v}{2 N}(1) \\
u=0,1, \cdots, M-1 ; \quad v=0,1, \cdots, N-1 ;
\end{gathered}
$$

In this formula:

$$
c(u)=\left\{\begin{array}{ll}
\sqrt{1 / M} & u=0 \\
\sqrt{2 / M} & u=1,2, \cdots, M-1
\end{array} \quad c(v)= \begin{cases}\sqrt{1 / N} & v=0 \\
\sqrt{2 / N} & v=1,2, \cdots, N-1\end{cases}\right.
$$

Two-dimensional discrete cosine transform (IDCT) using the following formula:

$$
\begin{gathered}
f(x, y)=\sum_{u=0}^{M-1} \sum_{v=0}^{N-1} c(u) c(v) F(u, v) \cos \frac{\pi(2 x+1) u}{2 M} \cos \frac{\pi(2 y+1) v}{2 N} \\
x=0,1, \cdots, M-1 ; \quad y=0,1, \cdots, N-1
\end{gathered}
$$

Where $\mathrm{x}, \mathrm{y}$ are spatial domain sample values ; and $\mathrm{u}, \mathrm{v}$ are the frequency-domain sample values. Digital images are usually expressed in square pixels, $\mathrm{M}=\mathrm{N}$ we can see from the above formula, the symbol coefficients of DCT relate to phase components.

\section{THE ALGORITHM}

We need to choose a texture image with black border as the original texture image. Add a black border is to ensure the conservation of energy in geometric transformations. The original texture images are described as: $F=\{f(i, j) \mid f(i, j) \in$ $\mathrm{R} ; 1 \leq \mathrm{i} \leq \mathrm{N} 1,1 \leq \mathrm{j} \leq \mathrm{N} 2\}, \mathrm{f}(\mathrm{i}, \mathrm{j})$ means the pixel gray value of the original texture image. In order to facilitate operation, we assumeN1=N2=N .

\section{A. Texture feature vector selection}

First, DCT transform the original texture image globally, DCT coefficient matrix obtained. Random selected seven lowfrequency coefficients in DCT coefficient matrix. "1" indicates a positive DCT coefficients (coefficients having a value of zero), and " 0 " indicates a negative coefficient. We can get the symbol sequence "1001010" as the image feature vectors. Observe in Table I we can find that the symbol sequence can be maintained similar to the original texture image whether the image through common attacks, geometric attacks or local nonlinear geometric attacks. As the same as the original texture image normalized correlation coefficient is 1.0. 
TABLE I. CHANGE OF DCT LOW-FREQUENCY COEFFICIENTS WITH RESPECT TO DIFFERENT ATTACKS

\begin{tabular}{|c|c|c|c|c|c|c|c|c|c|c|}
\hline Image Operation & $\operatorname{PSNR}(\mathrm{dB})$ & $\mathbf{F}(\mathbf{1 , 1})$ & $\mathbf{F}(1,2)$ & $\mathbf{F}(\mathbf{1}, \mathbf{3})$ & $\mathbf{F}(1,4)$ & $\mathbf{F}(\mathbf{1 , 5})$ & $F(1,6)$ & $\mathbf{F}(1,7)$ & $\begin{array}{c}\text { Coefficient symbol } \\
\text { Sequence }\end{array}$ & $\begin{array}{l}\text { Related } \\
\text { Degree }\end{array}$ \\
\hline Original image & 42.14 & 73.69 & -0.52 & -25.34 & 0.26 & -19.79 & 0.48 & -11.69 & 1001010 & 1.0 \\
\hline JEPG compression (5\%) & 23.00 & 75.05 & -0.65 & -25.29 & 0.38 & -19.96 & 0.60 & -11.65 & 1001010 & 1.0 \\
\hline Gaussian noise(3\%) & 18.02 & 73.03 & -0.53 & -22.59 & 0.11 & -17.65 & 0.46 & -10.46 & 1001010 & 1.0 \\
\hline Median filter[3x3] & 23.43 & 74.73 & -0.33 & -25.88 & 0.25 & -20.08 & 0.24 & -11.90 & 1001010 & 1.0 \\
\hline Translation(5pix) & 11.55 & 66.56 & -0.77 & -24.09 & 0.45 & -18.97 & 0.61 & -11.21 & 1001010 & 1.0 \\
\hline Rotation $\left(\right.$ clockwise $\left.5^{\circ}\right)$ & 13.32 & 73.53 & -0.57 & -25.76 & 0.26 & -19.47 & 0.35 & -10.78 & 1001010 & 1.0 \\
\hline Cropping(4\% from y) & 13.74 & 63.89 & -0.82 & -23.11 & 0.49 & -18.23 & 0.62 & -10.74 & 1001010 & 1.0 \\
\hline Scaling $(0.5)$ & & 36.84 & -0.26 & -12.67 & 0.13 & -9.90 & 0.24 & -5.85 & 1001010 & 1.0 \\
\hline Pinch distortion(30\%) & 15.59 & 68.21 & -0.51 & -24.80 & 0.49 & -20.19 & 0.45 & -10.50 & 1001010 & 1.0 \\
\hline Ripple distortion(100\%) & 17.91 & 70.68 & -0.61 & -24.86 & 0.29 & -19.33 & 0.72 & -11.23 & 1001010 & 1.0 \\
\hline Spherical distortion $(20 \%)$ & 14.16 & 75.02 & -0.85 & -24.17 & 0.23 & -18.46 & 0.61 & -11.95 & 1001010 & 1.0 \\
\hline Twirl distortion $\left(30^{\circ}\right)$ & 19.07 & 70.66 & -0.71 & -24.83 & 0.50 & -19.43 & 0.38 & -11.36 & 1001010 & 1.0 \\
\hline Wave distortion $(2 \%)$ & 21.41 & 70.65 & -0.47 & -24.79 & 0.57 & -19.50 & 0.49 & -11.27 & 1001010 & 1.0 \\
\hline Random wave distortion(Triangle) & 12.04 & 70.80 & -0.59 & -24.36 & 0.31 & -18.13 & 0.54 & -10.04 & 1001010 & 1.0 \\
\hline
\end{tabular}

It can be seen in Table II, the sequence of different texture images symbol are different. The correlation is small and less than 0.5. This shows that the symbols of DCT coefficient sequence can reflect the main features of the visual texture image. The vectors are essentially the same when texture images through a certain degree of common attacks, geometric attacks and local nonlinear geometric attacks. Therefore, the symbol of DCT coefficients sequence can be used as a texture image eigenvectors.

TABLE II. DIFFERENT TEXTURE IMAGE FEATURE VECTOR CORRELATION COEFFICIENT (VECTOR LENGTH 32BIT)

\begin{tabular}{|c|c|c|c|c|c|c|c|c|}
\hline & Sa & Sb & Sc & Sd & Se & Sf & Sg & Sh \\
\hline Sa & 1.00 & -0.08 & 0.10 & -0.06 & 0.06 & -0.09 & 0.13 & -0.01 \\
\hline $\mathrm{Sb}$ & -0.08 & 1.00 & -0.01 & 0.01 & 0.03 & 0.00 & 0.08 & 0.02 \\
\hline $\mathrm{Sc}$ & 0.10 & -0.01 & 1.00 & 0.08 & 0.08 & -0.02 & -0.05 & 0.09 \\
\hline $\mathrm{Sd}$ & -0.06 & 0.01 & 0.08 & 1.00 & -0.01 & 0.00 & -0.01 & 0.08 \\
\hline $\mathrm{Se}$ & 0.06 & 0.03 & 0.08 & -0.01 & 1.00 & 0.13 & -0.09 & 0.05 \\
\hline $\mathrm{Sf}$ & -0.09 & 0.00 & -0.02 & 0.00 & 0.13 & 1.00 & 0.04 & 0.11 \\
\hline $\mathrm{Sg}$ & 0.13 & 0.08 & -0.05 & -0.01 & -0.09 & 0.04 & 1.00 & 0.06 \\
\hline $\mathrm{Sh}$ & -0.01 & 0.02 & 0.09 & 0.08 & 0.05 & 0.11 & 0.06 & 1.00 \\
\hline
\end{tabular}

\section{B. Establish database of the texture feature vector}

1) Get the feature vector of the original security image using DCT

All original security pictures DCT transform and obtain DCT coefficient matrix FD (i, j). Random select $7 \times 7$ transform coefficients FD7 (i, j) and then obtain the visual feature vector $\mathrm{V}$ (j) by DCT coefficients symbolic computation.

$$
\begin{aligned}
& F D(i, j)=D C T 2(F(i, j) \\
& V(j)=-\operatorname{Sign}(F D(i, j))
\end{aligned}
$$

2) Store the original texture image's feature vector

3) Intelligent identification texture anti-counterfeiting

Step1: Get the feature vector of the tested texture image using DCT

Suppose the texture image to be $F^{\prime}(i, j)$, then we use DCT to the tested texture images and obtain DCT coefficient matrix
F '(i, j). Establish the database according to the method described above and obtain visual texture feature vector $\mathrm{V}$ '(j) of the tested image.

$$
\begin{aligned}
& F^{\prime}(i, j)=\operatorname{DCT} 2\left(F^{\prime}(i, j)\right) \\
& V^{\prime}(j)=\operatorname{Sign}\left(F^{\prime}(i, j)\right.
\end{aligned}
$$

Step2: Obtain the PSNR of the image in order to assess the quality of texture images after attacked.

Assuming the pixel value at each point of the image is I (i, $\mathrm{j}$ ), and the average value of pixels of the image is $\Gamma(i, j)$. M is the length of the texture image, $\mathrm{N}$ is the width of the texture image. PSNR is defined as:

$$
P S N R=10 \lg \left[\frac{M N \max _{i, j}(\mathrm{I}(i, j))^{2}}{\sum_{i} \sum_{j}\left(\mathrm{I}(i, j)-\mathrm{I}^{\prime}(i, j)\right)^{2}}\right]
$$

Step3: Compare NC of the original texture image with the tested texture image's NC. Defined as:

$$
N C=\frac{\sum_{j} V(j) V^{\prime}(j)}{\sum_{j} V^{2}(j)}
$$

The larger the value of $\mathrm{NC}$ is, the more approximation between the tested texture image $F$ ' $(i, j)$ and the original texture image $F(i, j)$. Determine whether it's original texture image or not by calculating NC.

Step4: Return the maximum value of NC to the user's phone.

\section{EXPERIENCE}

In our experiments, we randomly select 1000 group of independent binary sequence (value of 1 or -1). Every sequence consists of 32 bits. We randomly select one group in the 1000 data set as the feature vector embedded (we chose the 500th group here). Here the size of the original texture image (a) is $128 \times 128$. The corresponding DCT coefficient matrix is $F D(i, j)$.We choose the low-frequency coefficients $Y(j)(1 \leq j \leq L)$. The first value $\mathrm{Y}$ (1) on behalf of the DC component of the 
image, and then arranged from low to high frequency order. We chose the low-frequency coefficients do $4 \times 8=32$ to be feature vector $\mathrm{V}$, le $\mathrm{L}=32$. Select the $\mathrm{DCT}$ coefficient matrix as $F D(i, j), 1 \leq i \leq 4,1 \leq j \leq 8$. Extraction algorithm to extract the $V$ ' and determine whether it's original texture image or not by calculating $\mathrm{NC}$ of $\mathrm{V}$ and $\mathrm{V}$ '.

Currently, the original texture image store in the database and each original texture image size is $10 \mathrm{~K}$, namely 10240 bytes. Therefore, the database can be reduced share of 2560 times, greatly saves storage space, but also improve the transmission rate that improves the identification rate.

In the simulation, PSNR is used to access the quality of the tested image. The larger PSNR values represent higher image quality. $\mathrm{NC}$ is used to access the results of similarity detection. It represents the original texture image if the value of $\mathrm{NC}$ is greater than 0.5 .

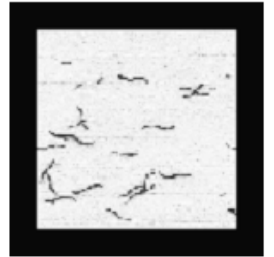

(a)original texture image

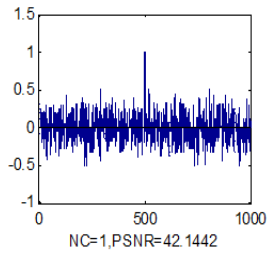

(b)Similarity detector
Fig. 1. Original texture image and detecting NC without attacks

Fig.1 (b) is the similarity detector. The similarity detect clearly, $\mathrm{NC}=1.0$. The original image is clearly detected.

\section{A. Common attacks}

\section{1) Adding Gaussian noise}

The texture image under Gaussian attacks $(3 \%)$ is shown in Fig.2 (a). The similarity can be detected as shown in Fig.2(b), NC=1.0. It proved that our proposed algorithm has strong robustness against noise attacks.

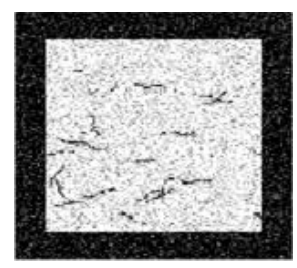

(a) Adding Gaussian noise

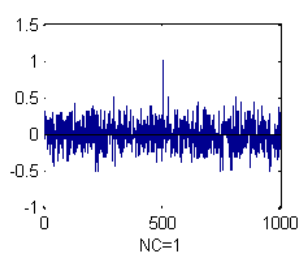

(b) Similarity detector
Fig. 2. Gaussian attack (3\%) of the texture image and the similarity detector

TABLE III. ANTI-GAUSSIAN NOISE INTERFERENCE TEST DATA

\begin{tabular}{|c|c|c|c|c|c|c|c|}
\hline Gaussian noise (\%) & $\mathbf{1}$ & $\mathbf{2}$ & $\mathbf{3}$ & $\mathbf{5}$ & $\mathbf{1 0}$ & $\mathbf{1 5}$ & $\mathbf{3 0}$ \\
\hline PSNR(dB) & 22.32 & 19.37 & 18.02 & 15.81 & 12.82 & 11.33 & 8.85 \\
\hline NC & 0.94 & 0.94 & 1.0 & 0.94 & 0.88 & 0.88 & 0.69 \\
\hline
\end{tabular}

The texture image PSNR drops to $8.85 \mathrm{~dB}$ when Gaussian noise strength of up to $30 \%$ can be seen from the experimental data. At this time, the $\mathrm{NC}=0.69$, but it still judged as original texture image by detecting. This shows that the use of the invention has better anti-Gaussian noise.

\section{2) JEPG compression}

Avoid combining SI and CGS units, such as current in amperes and magnetic field in oersteds. This often leads to confusion because equations do not balance dimensionally. If you must use mixed units, clearly state the units for each quantity that you use in an equation.

Fig.3(a) is a compressed mass of $5 \%$ of the image and the figure has appeared blocking effect. Fig.3(b) is the similarity detection, $\mathrm{NC}=0.94$.

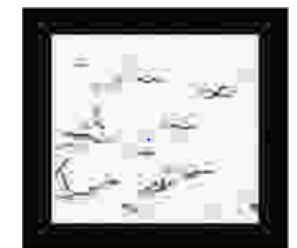

(a) JPEG compression (5\%)

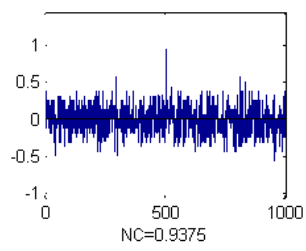

(b) Similarity detector
Fig. 3. JPEG (5\%) of the texture image and the similarity detector

TABLE IV. ANTI-JPEG COMPRESSION TEST DATA

\begin{tabular}{|c|c|c|c|c|c|c|}
\hline JEPG compression $(\boldsymbol{\%})$ & $\mathbf{2}$ & $\mathbf{5}$ & $\mathbf{1 0}$ & $\mathbf{2 0}$ & $\mathbf{3 0}$ & $\mathbf{4 0}$ \\
\hline PSNR $(\mathrm{dB})$ & 21.58 & 23.00 & 24.85 & 26.91 & 28.55 & 29.46 \\
\hline $\mathrm{NC}$ & 0.88 & 0.94 & 0.94 & 0.94 & 1.0 & 0.94 \\
\hline
\end{tabular}

The experimental data in Table IV is anti-JPEG compression. It still judged as the original texture image when the compression quality is $2 \%, \mathrm{NC}=0.88$. It proved that our proposed algorithm has strong robustness against JEPG compression attacks.

\section{B. Geometrical attacks}

\section{1) Rotation attacks}

Fig.4 (a) is the texture of the image rotation is $5^{\circ}$, PSNR $=13.32 \mathrm{~dB}$. Fig. 4 (b) is the similarity detection, the detection can be clearly judged as the original texture images, $\mathrm{NC}=$ 0.83 .

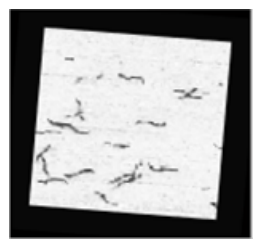

(a) Rotation attack

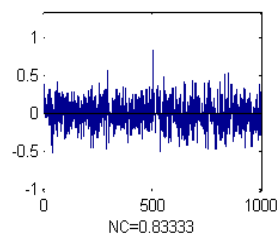

(b) Similarity detector
Fig. 4. Rotation attacks $\left(5^{\circ}\right)$ of the texture image and the similarity detector

TABLE V. EXPERIMENTAL DATA OF TEXTURE IMAGE ANTI-ROTATION

\begin{tabular}{|c|c|c|c|c|c|c|c|c|c|}
\hline Rotation angle & $\mathbf{0}^{\circ}$ & $\mathbf{1}^{\circ}$ & $\mathbf{2}^{\circ}$ & $\mathbf{3}^{\circ}$ & $\mathbf{4}^{\circ}$ & $\mathbf{5}^{\circ}$ & $\mathbf{6}^{\circ}$ & $\mathbf{7}^{\circ}$ & $\mathbf{1 0}^{\circ}$ \\
\hline PSNR $(\mathrm{dB})$ & 42.14 & 21.05 & 17.00 & 15.27 & 14.14 & 13.32 & 12.68 & 12.15 & 11.91 \\
\hline NC & 1.00 & 0.88 & 0.88 & 0.83 & 0.83 & 0.83 & 0.83 & 0.75 & 0.75 \\
\hline
\end{tabular}


Table $\mathrm{V}$ is the texture image anti-rotation attack experimental data. $\mathrm{NC}=0.75$ when the texture image is rotated $10^{\circ}$ and it still can be judged as the original texture image.

\section{2) Scaling attacks}

Fig.5(b) is a scaling factor of 0.5 for the texture image, then the center of the image is smaller than the original image; Fig.5(b) is a similarity detection, $\mathrm{NC}=1.00$, it can be determined to be the original texture image.

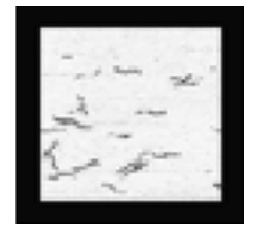

(a) scaling attack

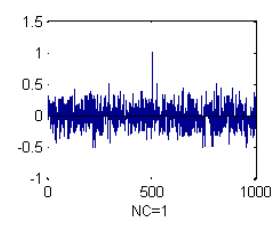

(b) Similarity detector
Fig. 5. Scaling attack $(\times 0.5)$ of the texture image and the similarity detector

TABLE VI. TEXTURE IMAGE SCALING ATTACK EXPERIMENTAL DATA

\begin{tabular}{|c|c|c|c|c|c|c|c|c|}
\hline Scaling factor & $\mathbf{0 . 2}$ & $\mathbf{0 . 5}$ & $\mathbf{0 . 6}$ & $\mathbf{0 . 7}$ & $\mathbf{0 . 8}$ & $\mathbf{1 . 1}$ & $\mathbf{1 . 5}$ & $\mathbf{2 . 0}$ \\
\hline $\mathrm{NC}$ & 0.56 & 1.00 & 0.94 & 0.81 & 0.81 & 0.94 & 1.00 & 1.00 \\
\hline
\end{tabular}

Table VI shows it can still be judged as the original texture image, indicating that the invention has strong anti-scaling capability.

\section{3) Pinch distortion attacks}

Fig.6 (a) shows the texture image under the pinch distortion $(30 \%)$ attacks, PSNR $=15.59 \mathrm{~dB}$. Fig.6 (b) is a similarity detection, it can be judged as the original texture image, $\mathrm{NC}=0.94$.

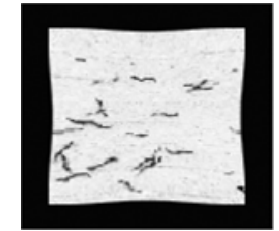

(a) pinch distortion attack

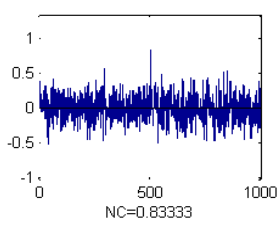

(b) Similarity detector
Fig. 6. Pinch distortion attack $(30 \%)$ of texture image and similarity detector

TABLE VII. EXPERIMENTAL DATA OF ANTI-EXTRUSION DISTORTION

\begin{tabular}{|c|c|c|c|c|c|c|c|}
\hline Pinch distortion $(\boldsymbol{\%})$ & $\mathbf{1 0}$ & $\mathbf{2 0}$ & $\mathbf{3 0}$ & $\mathbf{4 0}$ & $\mathbf{5 0}$ & $\mathbf{6 0}$ & $\mathbf{7 0}$ \\
\hline PSNR $(\mathrm{dB})$ & 20.17 & 17.15 & 15.59 & 14.42 & 13.39 & 12.48 & 11.63 \\
\hline NC & 1.00 & 0.94 & 0.94 & 0.88 & 0.88 & 0.81 & 0.63 \\
\hline
\end{tabular}

under the pinch distortion $(70 \%), \mathrm{NC}=0.63$, it still can be judged as the original texture image. The data show that the scheme is robust against pinch distortion attacks.

\section{CONCLUSION}

We propose an algorithm based on DCT in order to identify intelligently. It combines the visual feature vector and database technology, and doesn't require the original texture image in the identification process. The experimental results show that the algorithm can resist certain degree of common attacks and geometric attacks. Moreover, we just need to store the feature vectors of the texture images with our proposed algorithm, which can save a lot of storage space. Therefore, this method has good utility in the actual application process.

\section{Acknowledgment}

The work is supported by the National Natural Science Foundation of China (No.61263033) and the Hainan Province Special projects in colleges and universities scientific research (Hnkyzx2014-02).

\section{REFERENCES}

[1] S H Choi and C H Poon, "An RFID-based anti-counterfeiting system," IAENG International Journal of Computer Science, vol. 35, pp. 1-12, 2008 .

[2] C.H. Jiang, "Analysis of texture anti-counterfeiting technology," China Anti-counterfeiting Report, pp 48-50,20012.

[3] M.F. Chen, "Method for discerning false from genuine,":US, 6623041 B1, Sep.2003.

[4] F. Li, "Textures anti-counterfeiting structure, logistics systems and methods combine with bar code and SMS query."China,Sep.2012.

[5] M.F. Chen,"Texture anti-counterfeiting phone automatically recognizes logo":China, Feb.2012.

[6] N Kaewkammerd, K R Rao, "Wavelet based watermarking detection using multiresolution image registration," TENCON 2000, Proceedings. IEEE, Kaula Lumpr, Malaysia, vol.2, Sept. 2000, pp.171-175.

[7] M Kutter, S K Bhattacharjee, T Ebrhimi, "Towards second generation watermarking schemes," Proc of 1999 International Conference on Image Processing, vol.3, 1999, pp.320-323.

[8] W Cheung, G Hamarneh, "N-sift: N-dimensional scale invariant feature transform for matching medical images," Biomedical Imaging: From Nano to Macro, 2007, ISBI 2007, 4th IEEE International Symposium on IEEE, 2007, pp.720-723.

[9] Y Ke, R Sukthankar, "PCA-SIFT: A more distinctive representation for local image descriptors," Computer Vision and Pattern Recognition, 2004, CVPR 2004, Proceedings of the 2004 IEEE Computer Society Conference on. IEEE, Vol. 2, 2004, pp.506-513.

[10] H Zhou, Y Yuan, C Shi, “Object tracking using SIFT features and mean shift," Computer Vision and Image Understanding, vol.113, no.3, 2009, pp.345-352.

[11] M Pal, G M Foody, "Feature selection for classification of hyperspectral data by SVM," Geoscience and Remote Sensing, IEEE Transactions on, vol.48, no.5,2010, pp. 2297-2307.

[12] J Yang, D Zhang, A F Frangi, et al, "Two-dimensional PCA: a new approach to appearance-based face representation and recognition," Pattern Analysis and Machine Intelligence, IEEE Transactions on, vol.26, no.1, 2004, pp.131-137. 